\title{
Population-Level Death Rates From Novel Coronavirus (COVID-19) in South Korea
}

Asia Pacific Journal of Public Health $1-4$ (C) $2021 \mathrm{APJPH}$ (C) (i)

Article reuse guidelines: sagepub.com/journals-permissions DOI: I0.II77/I0I053952/993670 journals.sagepub.com/home/aph (S)AGE

\author{
Samir Soneji, $\mathbf{P h D}{ }^{1}(\mathbb{D}$, Hiram Beltrán-Sánchez, PhD², \\ JaeWon Yang, BA ${ }^{3}$, and Caroline Mann, BS'
}

\begin{abstract}
South Korea was among the first countries to report a case of the novel coronavirus (COVID-I9). As of August I4, 2020, South Korea reported 14873 confirmed cases and 305 deaths from COVID-19. We collected the number of laboratoryconfirmed cases and deaths by age from the Korean Centers for Disease Control and Prevention. We estimated populationlevel death rates by fitting a logistic regression model using Firth's bias reduction method. We calculated the age-standardized death rate (ASDR) varying the COVID-19 prevalence and level of death undercount. Estimated population-level death rates of COVID-19 (deaths per 100000 person-years) increased with age: 0.1 among 30 to 39 year olds, I.I among 60 to 69 year olds, and 14.5 among $\geq 80$ year olds. The ASDR (deaths per 100000 person-years) was 0.9 based on the current deaths and population-level prevalence of $0.03 \%$. If the prevalence increased to $0.6 \%$ and $30 \%$ of COVID-19 deaths had not been reported, the ASDR would further increase to I3.7 to become the seventh leading cause of death. Currently, the population-level mortality burden of COVID-19 in South Korea, as measured by the ASDR, was relatively low compared with other causes but could increase substantially if the prevalence increases from another outbreak or COVID-19 deaths were undercounted.
\end{abstract}

\section{Keywords}

COVID-19, coronavirus, South Korea, death rates

\section{What We Already Know}

South Korea was one of the first countries outside of China to report a case of the novel coronavirus (COVID-19). As August 14, 2020, South Korea reported 14873 cases (population-level prevalence of $0.03 \%$ ) and 305 deaths.

\section{What This Article Adds}

We estimated the population-level death rate using total confirmed cases and death counts. The death rate (deaths per 100 000 person-years) increased exponentially with age: from 0.1 among 30 to 39 year olds to 1.1 among 60 to 69 year olds to 14.5 among $\geq 80$ year olds. Overall, the age-standardized death rate (ASDR) of COVID-19 in South Korea was low (0.9 deaths per 100000$)$ and fell below the leading causes of death. The ASDR was likely low because of successful public health measures including containment, mitigation, and widespread testing. If, however, the prevalence of COVID19 increased to higher levels because of future outbreaks or if deaths have been undercounted, the mortality burden could increase substantially and exceed many leading causes of death.

\section{Introduction}

In response to the rapidly growing number of coronavirus disease 2019 (COVID-19) cases, South Korea raised the national alert level to the highest possible level, instituted voluntary lockdown of affected cities and provinces, and implemented widespread screening. ${ }^{1,2}$ Case fatality rates (ratio of deaths to number infected) are the most common measure used to assess COVID-19 mortality burden. However, comparison of case fatality rates across countries

\footnotetext{
'University of North Carolina at Chapel Hill, NC, USA

2University of California, Los Angeles, CA, USA

${ }^{3}$ Brown University, Providence, RI, USA

Corresponding Author:

Samir Soneji, University of North Carolina at Chapel Hill, Rosenau Hall, I 35 Dauer Drive, Chapel Hill, NC 27599, USA.

Email: sonejis@email.unc.edu
} 
is problematic because the true number of cases may far exceed the reported number of cases due to lack of testing. ${ }^{3}$ Additionally, COVID-19 is more fatal among older adults, and case fatality rates fail to account for age distribution that differ considerably between countries..$^{4-6}$

To address this research gap, we utilize standard demographic and epidemiological principles to estimate the agespecific COVID-19 death rate in South Korea. These death rates can then be summarized into the age-standardized death rate (ASDR), which accounts for differences in population age distributions and allows appropriate comparison across countries.

\section{Methods}

We began with the total number of COVID-19 cases and deaths by age group reported by South Korea to calculate case fatality rates and population-level prevalence. ${ }^{5,7}$ To estimate death rates by age, we fit a weighted logistic regression model using the penalized maximum likelihood method, which accounts for possible coefficient estimate bias from the small absolute number of deaths (especially among younger age groups). ${ }^{8-10}$ Estimated death rates were based on 207 days of exposure (January 21, 2020, to August 14, 2020). We annualized the estimated death rates by multiplying them by the inverse of the fraction of the year represented by the exposure period. We then calculated the ASDR for COVID19, with the age standard based on the 2016 South Korean population (most recent year for which the ASDRs of the leading causes of death was published). ${ }^{11}$

We fit a similar weighted logistic regression model to estimate elasticities between (1) prevalence and death rates and (2) case fatality and death rates. Covariates were the natural logarithm of prevalence and natural logarithm of case fatality rates, both by age group. We considered alternative scenarios in which the (1) prevalence of COVID-19 increased across age groups and (2) COVID-19 death counts were underreported. For each scenario, we adjusted the estimated death rates by alternative prevalence rates and undercount percentages. We estimated the COVID-19 ASDR for each scenario and compared it with leading causes of death (see the appendix; available online). ${ }^{11}$

\section{Results}

The first laboratory-confirmed COVID-19 case in South Korea occurred on January 21, 2020 (Supplemental Figure 1; available online). As of August 14, 2020 (207 days later), South Korea reported 14873 cases (population-level prevalence of $0.03 \%$ ) and 305 deaths. The case fatality rate increased from $0.1 \%$ among 30 to 39 year olds to $25.0 \%$ among $\geq 80$ year olds.

The estimated population-level death rate (deaths per 100 000 person-years) increased with age: 0.0 among 0 to 9 year

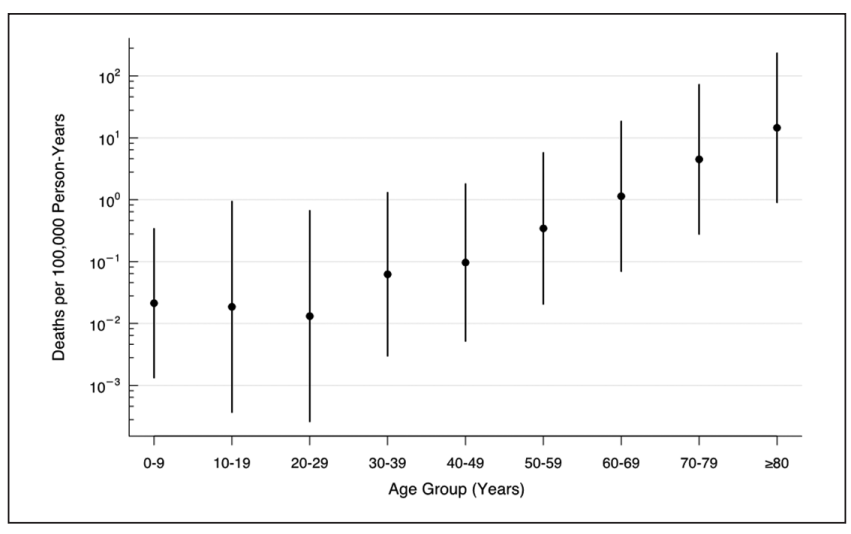

Figure I. Population-level mortality rates for COVID-19, South Korea (as of May 20, 2020).

Note: Vertical bars correspond to $95 \%$ confidence intervals estimated from a weighted logistic regression model using the penalized maximum likelihood method (see Methods section).

olds, 0.1 among 40 to 49 year olds, and 14.5 among $\geq 80$ year olds (Figure 1). The ASDR for COVID-19 was 0.9 deaths per 100000 person-years and fell below the 10 leading causes of death (Figure 2). We estimated the elasticity between prevalence and deaths to equal 0.6 ; a $1 \%$ relative increase in the prevalence across all age groups was associated with a $0.6 \%$ increase in death counts and the death rate (Supplemental Table 2; available online).

The mortality burden of COVID-19, as measured by the ASDR, could increase substantially if either the number of COVID-19 deaths was underreported or the prevalence increases (Figure 2). If prevalence increased from $0.03 \%$ to $0.6 \%$, the ASDR would increase from 0.9 to 10.0 deaths per 100000 person-years and approximately equal that of traffic accidents to become the 10th leading cause of death. If the prevalence increased to $3.4 \%$, the ASDR would increase to approximately the level of heart disease to become the second leading cause of death.

If the number of COVID-19 deaths has been underreported, smaller increases in the prevalence would lead to the disease becoming a leading cause of death. For example, if an additional $25 \%$ of COVID-19 deaths were not reported as decedents were not tested postmortem and prevalence increased from $0.03 \%$ to $0.6 \%$, the ASDR would increase to the level of chronic lower respiratory tract disease (seventh leading cause of death). If the prevalence increased to $2.7 \%$, the ASDR would increase to the level of heart disease (second leading cause of death).

\section{Discussion}

The COVID-19 mortality burden depends on 3 broad factors: (1) the overall health of the population prior to the epidemic, (2) the prevalence of COVID-19, and (3) the lethality of COVID-19 infection. The second and third factors likely 


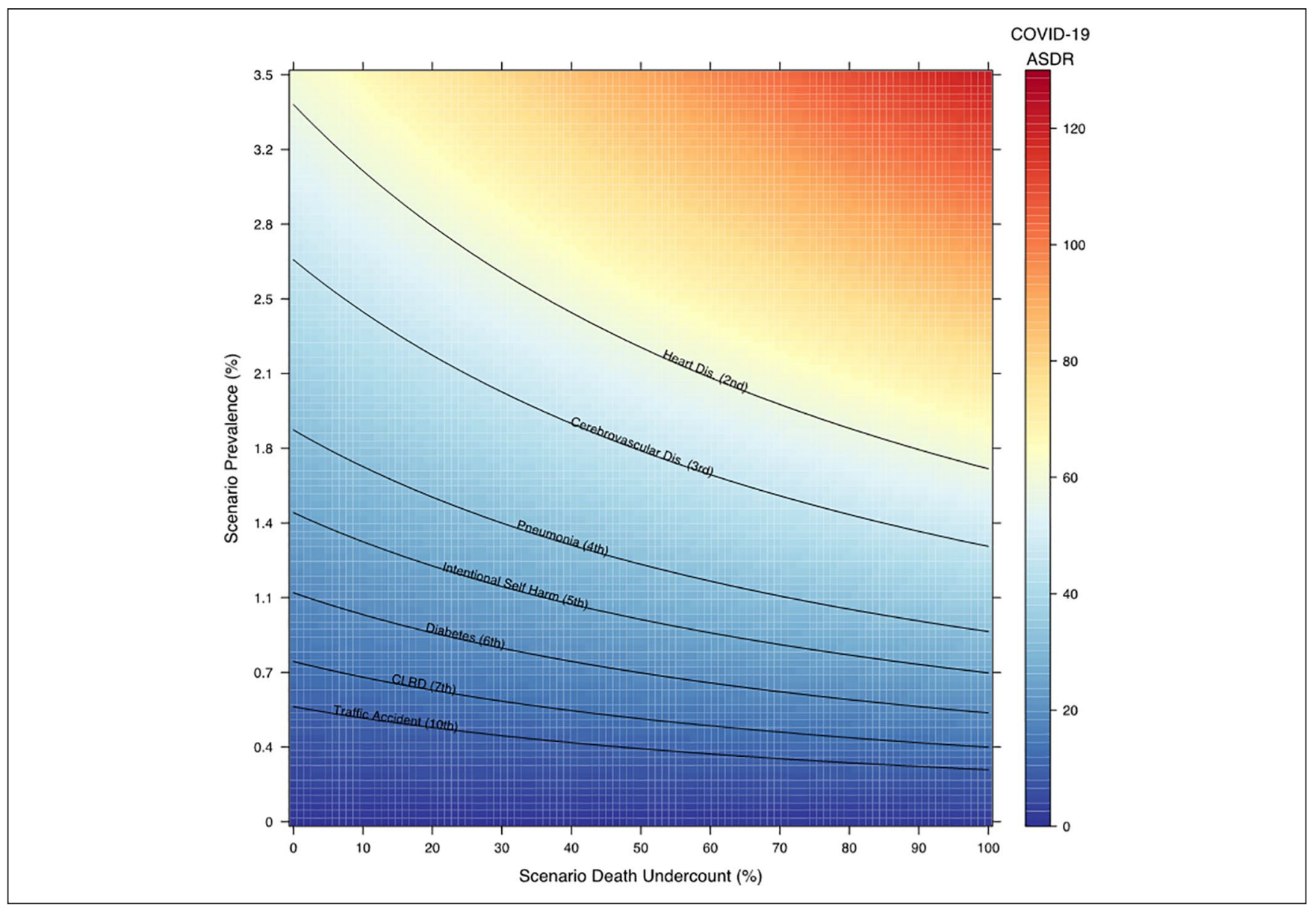

Figure 2. Age-standardized death rate of COVID-19 varying prevalence and undercount in deaths. Source: Authors' calculation.

contributed more to the relatively low mortality burden in South Korea compared with countries with higher number of deaths (eg, France, Italy, and Spain). With regard to the first factor, the overall health of the South Korean population was similar to that of the French, Italian, and Spanish populations prior to the pandemic, as measured by life expectancy at birth and ASDR from other respiratory diseases. ${ }^{12}$ With regard to the second factor, the prevalence of COVID-19 has remained comparatively low in South Korea because of successful containment and mitigation measures. These public health efforts have reduced the likelihood of transmission to individuals with comorbidities who are more likely than healthier individuals to be hospitalized, admitted to the intensive care unit, and die. ${ }^{13} \mathrm{With}$ regard to the third broad factor, COVID-19 infection has also proven less lethal among the elderly in South Korea than in Italy. For example, the case fatality rate among $\geq 80$ year olds equaled $25.9 \%$ in South Korea and $29.1 \%$ in Italy. However, rates could increase if the health care system becomes overwhelmed. ${ }^{14}$ Our approach enables researchers to estimate the COVID-19 ASDR based on country-specific prevalence and age-specific COVID-19 death rates, as well as account for differences in age distributions.

COVID-19 currently yields a relatively low mortality burden in South Korea compared with other causes of death because of its low prevalence. If the prevalence increases because of another outbreak or death counts have been underreported, the mortality burden could be substantially higher and exceed leading causes of death.

\section{Declaration of Conflicting Interests}

The author(s) declared no potential conflicts of interest with respect to the research, authorship, and/or publication of this article.

\section{Funding}

The author(s) received no financial support for the research, authorship, and/or publication of this article.

\section{ORCID iD}

Samir Soneji (iD https://orcid.org/0000-0002-0521-0707 


\section{Supplemental Material}

Supplemental material for this article is available online.

\section{References}

1. Cho J, Lee HK. South Korean president declares "war" on COVID-19 as deaths there reach 32. ABC News. Published March 3, 2020. Accessed March 19, 2020. https://abcnews. go.com/International/south-korean-president-declares-warcovid-19-deaths/story?id=69360757

2. Bicker L. Coronavirus in South Korea: how "trace, test and treat" may be saving lives. BBC News. Published March 12, 2020. Accessed March 19, 2020. https://www.bbc.com/news/ world-asia-51836898

3. Thomas K. As coronavirus testing increases, some labs fear a shortage of other supplies. The New York Times. Published March 11, 2020. Accessed March 19, 2020. https://www.nytimes. com/2020/03/11/health/coronavirus-testing-shortages.html

4. CDC COVID-19 Response Team. Severe outcomes among patients with coronavirus disease 2019 (COVID-19) - United States, February 12-March 16, 2020. MMWR Morb Mortal Wkly Rep. 2020;69:343-346. doi:10.15585/mmwr.mm6912e2

5. KCDC. Coronavirus infectious disease-19. Accessed April 9, 2020. http://www.cdc.go.kr

6. Task Force COVID-19 del Dipartimento Malattie Infettive e Servizio di Informatica. Sorveglianza Integrata COVID19 in Italia. Published April 6, 2020. Accessed January 25, 2021. https://www.epicentro.iss.it/coronavirus/bollettino/ Infografica_6aprile\%20ITA.pdf
7. World Health Organization. Coronavirus disease 2019 (COVID-19): situation report-58. Published March 18, 2020. Accessed January 25, 2021. https:/www.who.int/docs/defaultsource/coronaviruse/situation-reports/20200318-sitrep-58-covid-19.pdf?sfvrsn=20876712_2

8. Heinze G. A comparative investigation of methods for logistic regression with separated or nearly separated data. Stat Med. 2006;25:4216-4226. doi:10.1002/sim.2687

9. Heinze G, Schemper M. A solution to the problem of separation in logistic regression. Stat Med. 2002;21:2409-2419. doi:10.1002/sim.1047

10. Firth D. Bias reduction of maximum likelihood estimates. Biometrika. 1993;80:27-38. doi:10.1093/biomet/80.1.27

11. Shin HY, Lee JY, Kim JE, et al. Cause-of-death statistics in 2016 in the Republic of Korea. J Korean Med Assoc. 2018;61:573. doi:10.5124/jkma.2018.61.9.573

12. Organisation for Economic Co-Operation and Development. Health status: causes of mortality. Published April 21, 2020. Accessed January 25, 2021. https://stats.oecd.org/index.aspx? queryid $=30115 \#$

13. CDC COVID-19 Response Team. Preliminary estimates of the prevalence of selected underlying health conditions among patients with coronavirus disease 2019United States, February 12-March 28, 2020. MMWR Morb Mortal Wkly Rep. 2020;69:382-386. doi:10.15585/mmwr. mm6913e2

14. BBC News. Japan doctors warn health system may "break down.” Published April 18, 2020. Accessed April 20, 2020. https://www.bbc.com/news/world-asia-52336388 